\title{
Construir Políticas Infantiles desde la Ciencia y la Mística
}

JORGE JIMÉNEZ DE LA J. ${ }^{1}$

1. Profesor Asociado, Departamento de Salud Pública. Facultad de Medicina, Pontificia Universidad Católica de Chile.

\section{Vivir para Contarlo}

Vivir un proceso social de alto impacto y conocer a sus protagonistas, compartir con ellos y participar de la construcción de las estrategias, así como comprobar sus resultados, es un privilegio notable. Esa ha sido mi experiencia con las políticas de salud infantil en los últimos cincuenta años. Tengo 67 años de edad, me recibí de médico en 1968, a los 42 años de médico sumo los siete de estudios y prácticas, más aquellos que conviví con mi padre, médico y salubrista también. Toda una vida.

Es muy hermoso poder estar en la primera fila de estas batallas sociales de signo positivo, ascendentes, trabajadas y dolorosas ocasionalmente, pero de éxito y alegrías al fin de cuentas. Son un encuentro entre la razón y la fe, entre la ciencia y la mística. El racionalismo tiene un componente de positivismo que privilegia la evidencia por encima de la creencia en los valores que aparecen como utópicos o lejanos, irrealizables e incluso negativos para el progreso de la humanidad. La fe y la mística pueden parecer ciegas o excesivamente voluntariosas frente a la dimensión de los problemas o la propia maldad, el egoísmo y la indiferencia de las personas, pero son sin duda el componente que saca adelante los proyectos creados con inteligencia y con método.

Es esa mezcla virtuosa de fe en la vida de los niños y su valor, con la capacidad de la razón y el método puestos al servicio de una causa lo que hace la diferencia. Nadie se opone a salvar vidas infantiles, hay un consenso primario en la sociedad, que no se encuentra en otros temas relevantes en el siglo XXI como las enfermedades crónicas ligadas a los hábitos y estilos de vida o aquellas de transmisión sexual. Uno de los pre-requisitos de una política pública es su consenso social o de una mayoría importante que la sostenga. Y dicho consenso se consigue mejor si hay personas inspiradas que promuevan las causas justas.

\section{Un hijo, un Libro, un Árbol}

La vivencia de un proceso como el que motiva a los pediatras de Chile, y debe haber seguro muchas otras experiencias parecidas en el mundo, genera el impulso a escribir, narrar y juntar en recuentos ordenados, los elementos de estos avances en la salud infantil. Ese fue mi caso cuando inicié en los noventa la recolección de antecedentes para escribir una evaluación de las políticas públicas en la infancia. La pretensión inicial, ilusa resultó al final, era la de hacer una evaluación sistemática, basada en evidencia, con muchos números y análisis estadístico adecuado. Poco a poco me dí cuenta que más allá de un buen recuento con reflexiones no pasaría. Y sería un libro, ojala centrado en la gran historia de la disminución de la mortalidad infantil en Chile.

Correspondencia a:

Dr. Jorge Jiménez de la Jara

E-mail: jjimenez@med.puc.cl 
Al mirar de cerca el cuadro "Velorio del Angelito" de Manuel Antonio Caro, colgado en el living de la residencia del embajador de Chile en Londres, pude entender el dramatismo que el evento de una muerte significaba en la cultura de una sociedad, al punto que generaba expresiones de arte. Recordé las canciones de Violeta Parra y salí a buscar en libros y anales los estudios sobre canto popular a lo divino y me encontré con mucha literatura, conmovedora expresión del dolor ante la muerte temprana e injusta de un niño. Reviví una vez más la muerte de mi hijo prematuro por una enfermedad hemolítica por Rh y en aquellos años, 1974, con un peso muy bajo al nacer (1 600 gramos). Para la época, como decíamos a los padres frente a la fatalidad, se hizo todo lo que se pudo. Sólo quedó un cajoncito blanco, un recuerdo amargo, un angelito más en el cielo, pero nuestro, más cercano y presente.

Seguí investigando para convencerme que sería más un testimonio documentado, un mirar mi propia vida a la luz de mi familia, de mis hijos, de mi vocación por la pediatría social, que una revisión sistemática de política. Así nació "Angelitos Salvados"*.

\section{Héroes, Mística y Ciencia}

En todas las grandes empresas de la humanidad hay héroes, campeones, líderes, inspiradores; y la generación de las políticas de salud infantil en Chile no es una excepción. Los héroes son personas, hombres o mujeres que tienen una fe profunda y fuerte en lo que creen es una misión trascendente. En caso de la pediatría sus nombres son conocidos aunque su obra esté menos nítida: Calvo Mackenna, Roberto del Río, Schwarzenberg, Ariztía, Baeza Goñi, Meneghello, más los sanitaristas con visión como Cruz Coke, Grossi, entre otros.

El caso de las políticas sociales para la infancia es el de una política de alto consenso. Tanto así que en la historia de Chile, encontramos un "Primer Congreso Nacional de Protección de la Infancia" en 1912, encabezado por el Presidente de la República y organizado por el insigne pediatra Luis Calvo Mackenna. Sin embargo, tener consenso no significa tener una política efectiva, hace falta mucho estudio y una gran dedicación al desarrollo e implementación de las estrategias mas plausibles.

Muchos eventos sucesivos, relatados en el libro fueron manifestaciones de una línea conductora de la política pública para la infancia. Los programas de alimentación suplementaria de la Gota de Leche, los de protección a los hijos de obreros en el Seguro Obrero Obligatorio, la ley de Madre y Niño de Cruz Coke en 1937, las iniciativas de protección de la infancia (PROTINFA), las unidades sanitarias vecinales, hasta llegar al Servicio Nacional de Salud en 1952 que propone como su primera tarea la madre y el niño y que se consolida con éxito en los años 70 .

Por ello, a través de la investigación histórica de la pediatría y las políticas para la infancia, se observan personajes y grupos de alta motivación y de gran impacto. Uno de ellos fue el de la escuela del Profesor Julio Meneghello, llamada de la "Pediatría Clínica y Social". Su grupo de clínicos y salubristas dejó una huella amplia y profunda en Chile y en la región latinoamericana. Muchos nombres quedaron prendidos a las políticas y el desarrollo de soluciones desde la ciencia y la mística por servir: Francisco Mardones, Jorge Rosselot, Enrique Fanta, Oscar Undurraga, Fernando Mönckeberg, Guillermo Repetto, Mafalda Rizzardini entre los históricos; Eliana Cerutti, Armando Díaz, Carlos Casar, Isidoro Horwitz, Mario Ferreiro entre los más contemporáneos.

\section{Expresiones de humanismo en la política y en la ciencia}

Con un claro sentido humanista, muy propio de los médicos con motivación social, Meneghello describe los postulados e intereses sobre problemas del grupo que encabezaba: más interés por lo importante que por lo raro, más interés por las personas que por los casos, tanto interés por la salud como por las enfermedades, tanto interés por la prevención como por la curación, tanto interés por los padres y la familia como por el niño.

\footnotetext{
*Jiménez de la Jara Jorge: Angelitos Salvados (2009) Uqbar Editores, 215 pp.
} 
El equipo de Meneghello desarrolló su acción a través de dos estructuras creadas para sus propósitos: el Centro de Adiestramiento e Investigación Materno Infantil (CAIMI, creado en 1957) y el Laboratorio de Investigaciones Pediátricas. En ambas estructuras prevalecían los principios de integración científicoasistencial, por los cuales un solo equipo humano compartía los recursos y motivaciones de la universidad - con sus tareas de investigación y docencia- con el servicio de salud y su misión asistencial frente al gran desafío de la supervivencia de los niños.

El CAIMI fue un proyecto que hoy llamaríamos "cuasi experimento de base poblacional", en el cual se definen los instrumentos de intervención en salud, se identifica y capta una población dentro de un territorio, se declaran metas de cobertura y se evalúan periódicamente las actividades y sus resultados. Su área de trabajo correspondía al Distrito 32 de la $7^{\text {a }}$ Circunscripción Electoral de Santiago. En el momento no se conocían las características demográficas de la población, pues el último censo era de 1952 y sus datos no eran confiables en ese nivel.

El equipo publica su primera evaluación a los pocos meses de entrar en funciones. Allí analiza sus actividades asistenciales en el Programa Materno Infantil, construido según normas del SNS de 1957, buscando cumplir una meta de cobertura de población que ellos 1laman "nivel útil" y que fijan en $60 \%$ de las madres embarazadas y de niños recién nacidos y lactantes (menores de dos años); sus resultados comprueban que llegaban a 55\% de las embarazadas, $52 \%$ de los recién nacidos y $75 \%$ de los lactantes, cifras que se comparaban favorablemente con consultorios vecinos de Santiago Centro de la época.

En materia de atención infantil, dan cuenta de que el $32,9 \%$ de las atenciones consiste en controles de salud; el resto corresponde a morbilidad. Estos controles del niño en sus primeros meses y años de vida eran registrados en una ficha sintética diseñada por el Departamento de Fomento de la Salud del SNS, que incluía una primera gráfica de crecimiento ponderal, más los hitos del desarrollo psicomotor del primer año, las inmunizaciones y la alimentación. Un dato valioso, aportado por estas investigaciones del grupo de Meneghello, dice que un $71 \%$ de sus niños bajo control estaba bien nutrido, mientras que el resto tenía distintas formas de desnutrición. Igualmente se informaba de las actividades de carácter educativo dirigidas a las madres, las cuales eran cotidianas y versaban sobre preparación de biberones, baño del lactante, prevención de enfermedades transmisibles, diarreas, accidentes y alimentación en el hogar.

La enfermería de terreno y las encuestas de tipo social eran los otros dos instrumentos de trabajo de la tarea asistencial. En este punto anotan los autores un concepto importante en el desarrollo de recursos humanos: "Tenemos pleno convencimiento de que, a lo largo del país, la auxiliar de enfermería debidamente capacitada es el eje, en lo que a personal se refiere, de la mayoría de las acciones de fomento y protección de la salud infantil, de ahí el especial acento con que tratamos en el centro de establecer un eficaz programa de adiestramiento para este tipo de funcionarios". Como se ve, la sustitución de funciones ya era asumida en el equipo de salud como una estrategia eficaz para mejorar los rendimientos de los programas en aplicación.

En cuanto a las actividades de docencia y adiestramiento, junto con reconocer que la oportunidad docente creaba mejores condiciones de trabajo que el promedio nacional, las consideran un espacio para "observar y realizar bajo adecuada supervisión las técnicas y procedimientos propios de un programa materno infantil”; y también: “... lo básico es despertar en quienes se encuentran en aprendizaje una nueva inquietud frente a estas acciones integrales de salud, que exigen imperativamente la compenetración de la filosofía de trabajo en equipo". Los alumnos eran estudiantes de medicina, enfermería, educación física y educación familiar de las universidades de Chile y Católica, además de los del SNS, que tenía escuelas para profesiones de la salud. Ya se utilizaban técnicas docentes y de investigación personal con asignación de cumplimiento de un número fijo de casos. Los talleres de discusión en grupos reducidos, así como las fichas bibliográficas y de casos clínicos en tarjetas 
especiales, fueron instrumentos de aprendizaje que hicieron escuela en este centro de adiestramiento. Cada alumno tenía la obligación de ejecutar ciertas tareas del programa de salud materno-infantil, como un medio de aprendizaje de técnicas concretas de trabajo médicosocial. Se les enviaba por grupos a terreno para encuestar y apreciar los problemas de salud de una familia y proponer soluciones en conjunto con sus profesores.

Desde sus inicios, el CAIMI tuvo un programa de investigación que incluía el análisis de los problemas técnicos y administrativos de mayor relieve en la atención materno-infantil. Los temas que el centro se dedicó a estudiar, desde un principio, fueron los siguientes: biodemografía del área de demostración, prevalencia de morbilidad de la población en control, evaluación del estado nutritivo en control prenatal e infantil, apreciación del estado psicomotor de la población infantil en control, estudio longitudinal del desarrollo físico, bioquímico, psíquico y emocional de los diversos períodos de la vida infantil, estudio inmunológico para comprobar la eficacia biológica del programa de vacunas vigente, investigación útil para definir científicamente las pautas dietéticas en distintas etapas de la vida, evaluación de las normas terapéuticas de la práctica habitual de la atención externa conjugando la eficacia con el costo, análisis exhaustivo de las condiciones socioeconómicas, culturales, de vivienda y saneamiento del medio en las familias en control; y correlación de estos resultados con los problemas de salud vigentes. Por otro lado, la evaluación de los resultados de las campañas de educación sanitaria y la ejecución de un programa efectivo de coordinación del control prenatal, así como un servicio obstétrico que permitiera la óptima atención integral de la embarazada, del recién nacido y de la puérpera, evaluación comparativa de los procedimientos vigentes para medir el rendimiento de un programa de salud materno-infantil y de las innovaciones sugeridas a título experimental.

Consta históricamente que esta larga lista de temas fue abordada íntegramente por el centro, con resultados tan sólidos que a partir de ellos, en la década de 1960, dos personajes notables, Jorge Rosselot Vicuña y Francisco Mardones Restat, impulsarían una verdadera revolución en la supervivencia infantil en nuestro país.

Esta aproximación a la investigación operacional en salud no existía en Chile, y tampoco se había dado en otras partes del mundo de una manera tan rigurosa y sistemática, tan relacionada con la realidad socioeconómica y tan profunda en sus contenidos y metodología. Mirar hacia afuera, hacia la comunidad, y salir a su encuentro en equipos coordinados entre la universidad y los servicios de salud, resultaron actitudes decisivas para avanzar en la lucha contra la mortalidad infantil en la época.

\section{Ciencia e Investigación: \\ La Razón sirve a la Mística}

Tener mística para enfrentar problemas sociales no basta. Es preciso poner la razón al servicio de ella. En el caso del grupo de Meneghello, sin ser él propiamente un investigador, hubo espacio para ampliar el método científico y realizar lo que hoy se llama "Investigación translacional", es decir conocer y reconocer los mecanismos que operan en los fenómenos biosociales, pero tratando de llegar a la acción e intervención lo antes posible.

Muchas de estas ideas y preguntas fueron puestas a prueba en el Laboratorio de Investigaciones Pediátricas, dirigido por los profesionales Herman Niemeyer, Francisco Beas y Fernando Mönckeberg. Ellos, junto a un equipo de jóvenes investigadores, inquirieron e investigaron las causas y los mecanismos fisiopatológicos de la diarrea infantil y la deshidratación, las causas y consecuencias de la desnutrición en el intestino y en las anemias, el crecimiento y el desarrollo neurológico de los niños y muchos otros temas relacionados con las enfermedades importantes de aquellos años. Pero no solo investigaban sobre los problemas sino que también sus soluciones. Del Laboratorio de Investigaciones Pediátricas salieron fórmulas para las mezclas hidratantes y los medios eficientes para administrarlas, como la hidratación oral por vía nasogástrica con sondas. Esta solución llegó incluso al mer- 
cado bajo el nombre de Silhidrol, fabricada y distribuida por el antiguo laboratorio Silesia. Lamentablemente, no tuvo el éxito que después tendrían las soluciones orales hidratantes de la UNICEF.

Este Laboratorio fue la base sobre la cual se construyó en la década de 1970 el Instituto de Nutrición y Tecnología de los Alimentos, INTA, organismo básico para el desarrollo de las ciencias aplicadas a la alimentación y sus consecuencias en nuestro país.

\section{Conclusiones}

He querido contar mis razones y hallazgos en este proceso de investigación y reflexión sobre las políticas de salud infantil en Chile. Ha sido una experiencia muy gratificante poder mirar y mirarse frente a un marco de referencia tan rico en gentes, problemas y soluciones, dolores y alegrías. Dicen que los pediatras somos así, altamente motivados y sociales. Parece que es cierto.

\section{A LOS AUTORES}

Comunicamos a los autores de los artículos presentados para publicación en Revista Chilena de Pediatría, que ha concluido exitosamente el Proyecto Editorial del Fondo de Publicación de Revistas Científicas CONICYT 2008-2009 "Revista Chilena de Pediatría: De las Publicaciones en Papel a un Proceso Editorial On-Line basado en Open Journal System". Por lo tanto, solicitamos a los autores de los artículos presentados a partir de ahora, que ingresen a www.revistachilenadepediatria.cl, se registren e identifiquen de acuerdo a las instrucciones, y procedan a ingresar su contribución al sistema editorial electrónico según se detalla.

La Sociedad Chilena de Pediatria y Revista Chilena de Pediatría se enorgullecen de poner a disposición de nuestros colaboradores esta moderna herramienta editorial, en forma pionera entre las publicaciones biomédicas de nuestro país.

El Editor 